\title{
Management of intentional overdose of low-molecular-weight heparin
}

\author{
Clara Lu MD, Mark A. Crowther MD MSc, Siraj Mithoowani MD MHPE
}

Cite as: CMAJ 2021 January 31;194:E122-5. doi: 10.1503/cmaj.211083

A 42-year-old man presented to the emergency department 2 hours after self-injecting 225000 units of dalteparin (15 prefilled 15000-unit vials) with intent to self-harm. The patient's medical history included depression, heterozygous factor $\mathrm{V}$ Leiden mutation and recurrent venous thromboembolism (VTE). Previously, he had switched among several anticoagulants owing to intolerance or breakthrough thrombosis, including warfarin, various preparations of low-molecular-weight heparin (LMWH) and, most recently, rivaroxaban. The injected dalteparin was left over from a previous prescription for 15000 units daily. He had taken his last dose of rivaroxaban 3 days before the current presentation.

The patient's vital signs were stable, and we observed no evidence of bleeding other than bruising around multiple abdominal injection sites. Initial blood work results showed hemoglobin of 121 (normal 130-180) g/L, creatinine of 99 (normal 60-110) $\mu \mathrm{mol} / \mathrm{L}$, estimated glomerular filtration rate (GFR) of 81 (normal $>60) \mathrm{mL} / \mathrm{min}$, international normalized ratio of 1.6 (normal $0.8-1.2$ ), activated partial thromboplastin time (aPTT) of $>150$ (normal 22-35) seconds, and LMWH anti-Xa level of 8.94 (therapeutic range $0.5-1.0$ ) $\mathrm{U} / \mathrm{mL}$. The patient's rivaroxaban level, measured by a drug-specific anti-Xa assay (Diagnostica Stago, Asnières sur Seine, France), was 351 (expected therapeutic range 182-408) ng/mL.

The internal medicine service admitted the patient, with support from the hematology and thrombosis service, and treated him with prophylactic intravenous protamine sulfate $50 \mathrm{mg}$ every 6 hours and oral tranexamic acid $500 \mathrm{mg}$ every 8 hours.

Eighteen hours after his overdose, the patient developed progressive left arm pain. He recalled no preceding trauma. Examination showed arm edema, antecubital fossa ecchymosis surrounding a venipuncture site and a weak radial pulse. The plastic surgery service measured the pressure in his left anterior arm compartment, finding it to be elevated at 30 (normal 0-8) $\mathrm{mm} \mathrm{Hg}$, confirming a diagnosis of anterior compartment syndrome, which we attributed to a hematoma after venipuncture, and subsequently performed an urgent bedside fasciotomy.

Figure 1 shows a timeline of clinical events, coagulation blood tests and treatment. During the period before and after the

\section{Key points}

- Bleeding in low-molecular-weight heparin (LMWH) overdose is unpredictable and may develop insidiously in deep tissues such as retroperitoneal and intramuscular spaces.

- Protamine sulfate partially reverses the anticoagulant effect of LMWH and may prevent bleeding from LMWH overdose.

- Serial monitoring of LMWH anti-Xa levels or activated partial thromboplastin time or both may help to guide therapy.

fasciotomy, the patient received three $50 \mathrm{mg}$ doses of protamine sulfate, with the third dose in response to bleeding from the surgical site, after which hemostasis occurred. Two days after fasciotomy, we started the patient on VTE prophylaxis with dalteparin 5000 units subcutaneous daily, given his high thrombotic risk. He underwent an uncomplicated wound closure 15 days later.

A family member returned unused LMWH vials for disposal. The patient described no further suicidal ideation and resumed rivaroxaban $20 \mathrm{mg}$ daily for thromboprophylaxis. After liaison with the psychiatry team, he was discharged to a mental health facility and followed in the outpatient thrombosis clinic with no long-term complications.

\section{Discussion}

\section{Epidemiology}

Although LMWH overdose is rare, heparin-associated bleeding is common. Our case illustrates an approach to managing LMWH overdose and provides practical lessons on the use of protamine sulfate to prevent and treat heparin-associated bleeding. Estimates suggest that heparin reversal with protamine sulfate is used in at least 2 million patients annually. ${ }^{1}$

We identified 10 case reports describing 16 episodes of LMWH overdose in adult patients since 2009. ${ }^{2-8}$ Although most of these overdoses were intentional, 2 were accidental, caused by iatrogenic miscalculation errors with multidose vials. ${ }^{2,3}$ We also identified a case series of 21 patients, but the authors reported little detail on management and included pediatric patients as well as patients with very mild overdoses. ${ }^{9}$ 


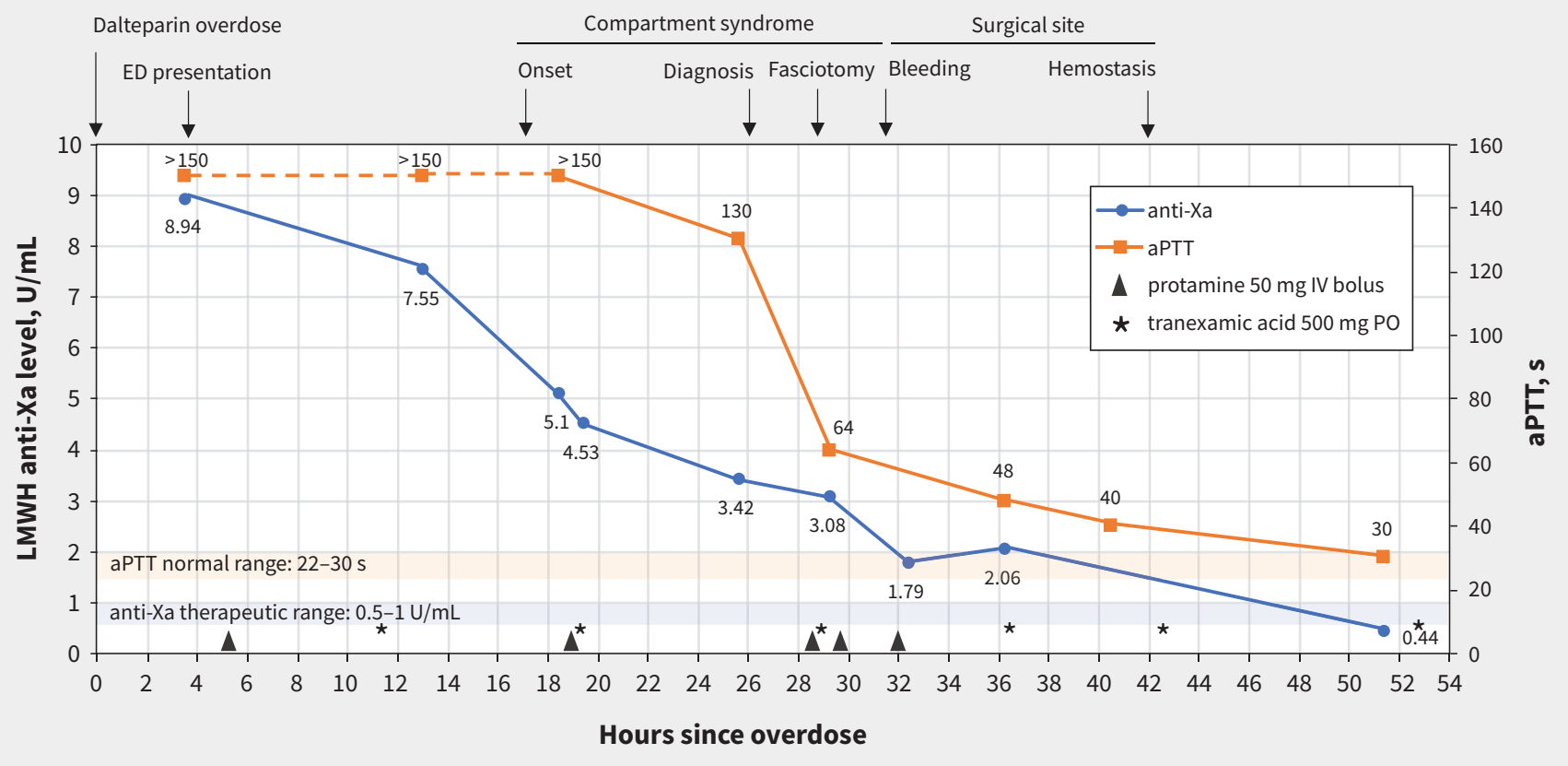

Figure 1: Clinical and biochemical timeline of overdose of low-molecular-weight heparin (LMWH). The orange line representing activated partial thromboplastin time (aPTT) is dashed at the beginning, as the first 3 aPTT values were reported as $>150 \mathrm{~s}$ (above the upper limit of measurement). Note: ED = emergency department, IV = intravenous, PO = orally.

\section{Protamine sulfate treatment}

Protamine sulfate forms a stable complex with heparin, neutralizing its anticoagulant effect. American College of Chest Physicians guidelines suggest using protamine sulfate to neutralize LMWH when necessary, although they give no specific recommendations on managing LMWH overdose. ${ }^{10}$ Among reported cases of LMWH overdose, protamine sulfate was administered prophylactically in 6 cases, therapeutically in 2 cases, and both prophylactically and therapeutically in 2 cases when bleeding developed during hospital stay, as occurred in our patient.

Our case illustrates 3 potential indications for protamine sulfate: to prevent bleeding after LMWH overdose, to treat intramuscular bleeding, and as prophylaxis before emergency fasciotomy.

Because the half-life of protamine sulfate is 7 minutes, its effect is transient unless continuously infused. This is illustrated by our patient, in whom LMWH anti-Xa levels decreased from $3.08 \mathrm{U} / \mathrm{mL}$ (before protamine sulfate) to $1.79 \mathrm{U} / \mathrm{mL}$ (after the final dose of protamine sulfate), but rose again to $2.06 \mathrm{U} / \mathrm{mL} 4$ hours later. Second, given concerns regarding hypotension and anaphylaxis, the product monograph recommends a maximum single dose of $50 \mathrm{mg}$ infused over a minimum of 10 minutes and cautious use in patients with fish allergy. ${ }^{11}$ However, the dose required to treat LMWH overdose (1 mg per 100 anti-Xa units, with $1 \mathrm{mg}$ of enoxaparin equal to 100 anti-Xa units) often far exceeds this threshold. No adverse effects from protamine sulfate were reported among previously published cases of LMWH overdose, even with continuous infusions and cumulative doses up to $500 \mathrm{mg.}{ }^{3,6}$ Third, although protamine sulfate fully neutralizes anti-lla activity attributable to LMWH, it neutralizes only $60 \%-74 \%$ of anti-Xa activity. $3,6,7$
Bleeding complications from LMWH overdose can be life threatening. Among 16 reported cases, 1 patient died after developing disseminated intravascular coagulation and multiorgan failure, ${ }^{4}$ and 5 cases resulted in major bleeding, including spontaneous retroperitoneal hematoma, ${ }^{5}$ hemothorax after cardiac surgery ${ }^{2}$ and traumatic compartment syndrome. ${ }^{6}$ This raises a key clinical question: should protamine sulfate be administered prophylactically for LMWH overdose or only when bleeding occurs? Our experience suggests that either use is reasonable, as bleeding complications may evolve rapidly and unpredictably.

Therapeutic adjuncts including tranexamic acid, ${ }^{2,6}$ recombinant factor VIIa, ${ }^{5}$ or other coagulation factor concentrates can be considered if bleeding is not mitigated by protamine sulfate. These therapies should be used judiciously as they are off label and some are associated with an increased risk of thromboembolism.

\section{Laboratory monitoring}

Serial monitoring of LMWH anti-Xa levels can help guide management in several ways. A baseline measurement can establish the severity of the overdose, particularly when there is renal impairment or when the history is limited or unreliable. ${ }^{3}$ Levels of LMWH anti-Xa activity can also help determine the efficacy of protamine sulfate if measured soon after administration, and guide its dosing, especially when administered as an IV infusion. ${ }^{3,6}$

Clinical correlation is required to interpret anticoagulant drug levels. Our patient's rivaroxaban level (measured by drugspecific anti-Xa assay) was likely confounded by the anti-Xa 
effect of LMWH. Levels of LMWH anti-Xa activity can be falsely elevated by other Factor Xa inhibitors, including rivaroxaban. Our patient's history, physical examination and response to protamine sulfate strongly suggested a LMWH overdose rather than rivaroxaban overdose.

The feasibility of anti-Xa monitoring depends on test availability and turnaround time. At our institution, turnaround time for a LMWH anti-Xa level ranges between 3 and 18 hours, depending on when the test is ordered. Therefore, we used the aPTT as an additional real-time marker of LMWH toxicity. The aPTT is normal or minimally prolonged at therapeutic doses of LMWH. At supratherapeutic LMWH doses, larger chain molecules within LMWH preparations potentiate thrombin inhibition, leading to marked aPTT prolongation. ${ }^{5}$ Our case mirrors previous observations that aPTT normalizes well before anti-Xa levels in LMWH overdose. ${ }^{5}$ Thirty-six hours after overdose, our patient's anti-Xa level remained substantially elevated $(2.06 \mathrm{U} / \mathrm{mL})$, whereas the aPTT was minimally prolonged $(48 \mathrm{~s})$.

Our case shows that if anti-Xa levels are unavailable, a prolonged aPTT may be used as a surrogate to detect severe LMWH toxicity. Prolongation of the aPTT can support the decision to continue administering protamine sulfate in the presence of bleeding, and normalization of the aPTT may be considered a threshold to discontinue prophylactic protamine sulfate in the absence of bleeding. Importantly, a normal aPTT cannot exclude the presence of LMWH, and anti-Xa levels remain the most useful measure. Tranexamic acid is an antifibrinolytic drug with no appreciable effect on aPTT or anti-Xa levels.

\section{Other management principles}

Box 1 summarizes key management principles. Patients with substantial overdose should be admitted to a monitored setting until aPTT or LMWH anti-Xa levels normalize. On admission, we suggest carefully securing intravenous access, ordering a group and screen and confirming local availability of protamine sulfate and other adjunctive therapies in case of severe bleeding. Clinicians should monitor patients closely for insidious bleeding into deep tissues (e.g., retroperitoneal and intramuscular spaces), dropping hemoglobin levels or renal dysfunction. Where available, poison control and hematology or thrombosis specialists can be consulted for further guidance, particularly for cases of massive overdose or severe bleeding. Clinicians should avoid excess vein trauma using measures similar to guideline recommendations for patients with hemophilia ${ }^{12}$ - for example, by selecting experienced staff for venipuncture and using small-gauge needles.

The decision to resume anticoagulation after overdose should be individualized depending on medical and psychiatric comorbidities. If concerned about recurrent overdose, clinicians may consider switching from LMWH to an oral anticoagulant for which there is a specific reversal agent (e.g., warfarin or dabigatran). Mental health support, supply limits on anticoagulant prescriptions, supervised administration and close follow-up can increase treatment safety. ${ }^{7}$ For patients who experience accidental overdoses, substituting multidose vials with prefilled syringes may prevent dosing errors. Anticoagulants from previous treatment courses should be returned for disposal.
Box 1: Management principles for overdose of lowmolecular-weight heparin

\section{Initial evaluation}

- Ensure hemodynamic stability and obtain intravenous access.

- Maintain a high index of suspicion for insidious bleeding into deep tissues.

\section{Management}

- Admit the patient to a monitored setting until coagulation parameters stabilize.

- Administer intravenous protamine sulfate (up to $1 \mathrm{mg}$ per 100 anti-Xa units) in repeated $50 \mathrm{mg}$ bolus doses or as an intravenous infusion to treat and possibly prevent bleeding.

- If bleeding occurs, address source control in addition to reversing low-molecular-weight heparin (LMWH).

- Contact Poison Control, particularly for cases of suspected or confirmed polysubstance overdose.

- Consider consulting the thrombosis or hematology service, particularly for cases involving massive overdose or severe bleeding.

\section{Laboratory monitoring}

- Order a complete blood count and serum creatinine with admission blood work to assess baseline hemoglobin and renal clearance.

- Serially monitor LMWH anti-Xa levels or activated partial thromboplastin time to assess response to protamine sulfate.

\section{References}

1. Carr JA, Silverman N. The heparin-protamine interaction. A review. J CardiovasC Surg (Torino) 1999;40:659-66.

2. Weber CF, Meybohm P, Kramer S, et al. Hemorrhage after accidental overdosage of enoxaparin: monitoring and therapy [article in German]. Anasthesiol Intensivmed Notfallmed Schmerzther 2014;49:368-73.

3. Al Saleh AS, Anderson D. Inadvertent overdose of low-molecular-weight heparin in an elderly patient with deep vein thrombosis and acute kidney injury. Can J Hosp Pharm 2016;69:320-2.

4. Schulz Y, Larsch K-P, Weller J-P, et al. Suizid durch subkutane Masseninjektion niedermolekularen Heparins. Rechtsmedizin 2009;19:95-101.

5. Byrne M, Zumberg M. Intentional low-molecular-weight heparin overdose: a case report and review. Blood Coagul Fibrinolysis 2012;23:772-4.

6. Odeberg J, Carlsson A, Hällsjö-Sander C, et al. A case series of five episodes of massive LMWH non-fatal self-induced overdose in a single patient. Thromb Res 2012;129:668-70.

7. Balla I, Karafotias I, Christopoulos C. Intentional overdose with tinzaparin: management dilemmas. J Emerg Med 2014;46:197-201.

8. Zhao J, Peiris M, Levin M. Intentional 24-fold overdose of low-molecular-weight heparin. Pathology 2020;52(Suppl 1):S117.

9. Ngo AS-Y, Tan D, Olson KR. Low molecular weight heparin overdose: a 10 year case series. Asia Pac J Med Toxicol 2013;2:68-70.

10. Garcia DA, Baglin TP, Weitz JI, et al. Parenteral anticoagulants: Antithrombotic Therapy and Prevention of Thrombosis, 9th ed: American College of Chest Physicians Evidence-Based Clinical Practice guidelines. Chest 2012;141(Suppl):e24S-43S.

11. Protamine sulfate injection, USP. Toronto: Fresenius Kabi Canada. Available: https://www.fresenius-kabi.com/en-ca/products/protamine-sulfate-injection -usp (accessed 2021 Jan. 3).

12. Srivastava A, Brewer AK, Mauser-Bunschoten EP, et al. Guidelines for the management of hemophilia. Haemophilia. 2013;19(1):e1-47. 
Competing interests: Mark Crowther reports receiving consulting fees from Servier Canada, Asahi Kasei, Precision Biologics and Hemostasis Reference Laboratories; payments or honoraria for educational material or presentations from Bayer, Pfizer, CSL Behring and Diagnostica Stago; and payment for expert testimony for various cases in Canada and the United States. Dr. Crowther also reports holding positions as Treasurer of the American Society of Hematology and The Anticoagulation Forum, and as Vice Chair of the Senior Research Committee, Heart and Stroke Foundation. Dr. Crowther has past and current relationships with a number of not-for-profit and for-profit entities that are not pharmaceutical manufacturers, and holds the Leo Pharma Chair in Thromboembolism Research at McMaster University. Dr. Siraj Mithoowani reports receiving support from Leo Pharma for attending a meeting. No other competing interests were declared.

This article has been peer reviewed.

The authors have obtained patient consent.

Affiliation: Department of Medicine, McMaster University, Hamilton, Ont.

Contributors: All of the authors contributed to the conception and design of the work, drafted the manuscript, revised it critically for important intellectual content, gave final approval of the version to be published and agreed to be accountable for all aspects of the work.

Content licence: This is an Open Access article distributed in accordance with the terms of the Creative Commons Attribution (CC BY-NC-ND 4.0) licence, which permits use, distribution and reproduction in any medium, provided that the original publication is properly cited, the use is noncommercial (i.e., research or educational use), and no modifications or adaptations are made. See: https://creativecommons. org/licenses/by-nc-nd/4.0/

Correspondence to: Siraj Mithoowani, siraj.mithoowani@medportal.ca 